\title{
SUBHARMONIC FUNCTIONS OF ORDER $r$
}

VICTOR L. SHAPIRO ${ }^{1}$

1. Given a domain $G$ in the plane, we shall say that $F(x, y)$ is subharmonic of order $r$ in $G$ if $F(x, y)$ is in class $C^{2(r-1)}$ and $\Delta^{r-1} F(x, y)$ is subharmonic in $G$, an analogous definition holding in $n$-dimensional space. When $r=1$, we see that this definition coincides with the definition of a continuous subharmonic function $\left(\Delta^{r}\right.$ standing for the Laplacian operator iterated $r$ times and coinciding with the identity operator when $r=0)$. We shall say that $F(x, y)$ has a generalized $r$ th Laplacian of the first or second kind at $\left(x_{0}, y_{0}\right)$ equal to $\alpha_{r}$ or $\beta_{r}$, respectively, according as

$$
\begin{aligned}
L\left(F ; x_{0}, y_{0} ; t\right) & \equiv \frac{1}{2 \pi} \int_{0}^{2 \pi} F\left(x_{0}+t \cos \theta, y_{0}+t \sin \theta\right) d \theta \\
& =\alpha_{0}+\alpha_{1} t^{2} /[2 !]^{2}+\cdots+\alpha_{r} t^{2 r} /[2 r r !]^{2}+o\left(t^{2 r}\right),
\end{aligned}
$$

or

$$
\begin{aligned}
A\left(F ; x_{0}, y_{0} ; t\right) \equiv & \frac{1}{\pi t^{2}} \int_{0}^{t} \rho d \rho\left[\int_{0}^{2 \pi} F\left(x_{0}+\rho \cos \theta, y_{0}+\rho \sin \theta\right) d \theta\right] \\
& =\beta_{0}+\beta_{1} t^{2} / 2[2 !]^{2}+\cdots \\
& +\beta_{r} t^{2 r} /(r+1)[2 r r !]^{2}+o\left(t^{2 r}\right),
\end{aligned}
$$

where $\alpha_{i}$ and $\beta_{i}(i=0, \cdots, r)$ are constants and $t$ tends to zero. In (1) it is assumed that $F(x, y)$ is integrable on the circumference of every circle of sufficiently small radius with center $\left(x_{0}, y_{0}\right)$, and in (2) it is assumed that there is a disc with center $\left(x_{0}, y_{0}\right)$ on which $F(x, y)$ is integrable.

Similarly, generalized $r$ th Laplacians of the first and second kind can be defined for $n$-dimensional space. (See $[5$, p. 261], where the expression for the generalized $m$ th Laplacian of the first kind can be obtained by replacing the last term on the right side of (39) by $o\left(R^{2 m}\right)$.) Designating the $n$-dimensional sphere of radius $t$ with center $P_{0}$ by $D\left(P_{0}, t\right)$ and assuming the integrability of $F(P)$ in every such sphere of sufficiently small radius, we then say that $F(P)$ has a generalized $r$ th Laplacian of the second kind at $P_{0}$ equal to $\beta_{r}$ if

Presented to the Society, October 24, 1953; received by the editors September 17, 1953 and, in revised form November 6, 1953.

1 This paper was done while the author was on a Rutgers University Research Council Grant. 


$$
\begin{aligned}
\frac{n \Gamma(n / 2)}{2 \pi^{n / 2} t^{n}} \int_{D\left(P_{0}, t\right)} F(P) d P & \\
& =n \Gamma(n / 2) \sum_{i=0}^{r} \frac{t^{2 i} \beta_{i}}{2^{2 i+1} i ! \Gamma(i+1+n / 2)}+o\left(t^{2 r}\right) .
\end{aligned}
$$

If $F(x, y)$ is in class $C^{(0)}$ in a domain $G$ and the generalized first Laplacian of the first or second kind is non-negative throughout $G$, then it is known $[8, \mathrm{p} .14]$ that $F(x, y)$ is subharmonic in $G$. It is the purpose of this paper to prove a similar result for subharmonic functions of order $r$. In particular, designating the generalized $r$ th Laplacians of the first and second kind by $\Delta_{1}^{r}$ and $\Delta_{2}^{r}$, respectively, we shall show that if $F(x, y)$ is in $C^{2(r-1)}$ and $\Delta_{2}^{r} F(x, y) \geqq 0$ in $G$ then $F(x, y)$ is subharmonic of order $r$. Using the anti-Laplacian operator for a bounded domain, we then apply this result to obtain a new sufficient condition for a function in $\operatorname{Lip} \alpha, \alpha>0$, to be subharmonic. We also give some applications to the theory of double trigonometric series.

Though the theorems on subharmonic functions of order $r$ are proven only for the plane, it will be clear from the nature of the proofs that analogous results hold in $n$-dimensional space.

2. Unless otherwise stated the notation for this paper will be that of Radó [8, in particular p. 3]. $\bar{D}$ will designate the closure of the set $D$. $D\left(x_{0}, y_{0} ; t\right)$ will represent the open disc with center $\left(x_{0}, y_{0}\right)$ and radius $t ; C\left(x_{0}, y_{0} ; t\right)$ will designate its circumference.

Replacing subharmonic by harmonic or superharmonic in the first sentence of $\$ 1$, we have the definition for $F(x, y)$ to be harmonic of order $r$ and superharmonic of order $r$ in $G$, respectively.

Given $f(x, y)$ bounded and continuous in a bounded domain $R$, we define the anti-Laplacian operator of $f$ in $R, \Delta^{-1} f(x, y)$, to be

$$
\begin{aligned}
& \Delta^{-1} f(x, y)=\frac{1}{2 \pi} \iint_{R} f(u, v) \log \rho d u d v \\
& \quad \text { where } \rho=\left[(x-u)^{2}+(y-v)^{2}\right]^{1 / 2} .
\end{aligned}
$$

$\Delta^{-r} f(x, y)$ is then defined as $\Delta^{-1}\left(\Delta^{-(r-1)} f(x, y)\right)$.

3. The main results of the paper are the following theorem and corollaries.

Theorem 1. Let $F(x, y)$ be in class $C^{2(r-1)}$ in a domain $G, r$ an integer $\geqq 1$, and let $E$ be a closed bounded set of capacity zero contained in $G$. Suppose $\Delta_{2}^{r} F(x, y) \geqq 0$ for $(x, y)$ in $G-E$. Then $F(x, y)$ is subharmonic of order $r$ in $G$. 
Corollary 1. Let $f(x, y)$ be in $\operatorname{Lip} \alpha, \alpha>0$, on the bounded domain $R$ and let $E$ be a closed bounded set of capacity zero contained in $R$. Suppose $\Delta_{2}^{2} \Delta^{-1} f(x, y) \geqq 0$ for $(x, y)$ in $G-E$. Then $f(x, y)$ is subharmonic in $R$.

Corollary 2. Let $F(x, y)$ be in class $C^{2(r-1)}$ on a domain $G$ and let $E_{1}$ be a closed bounded set of capacity zero (not necessarily contained in $G$ ). Suppose $\Delta_{2}^{r} F(x, y)=0$ in $G-E_{1} G$. Then $F(x, y)$ is harmonic of order $r$ in $G$. Furthermore for $r \geqq 2$ the hypothesis concerning class $C^{2(r-1)}$ cannot be weakened to class $C^{2(r-1)-1}$.

The results are stated in terms of $\Delta_{2}^{r}$, but since $F(x, y)$ and $\Delta^{-1} f(x, y)$ are continuous, they are, a fortiori, true if stated in terms of $\Delta_{1}^{r}$.

Corollary 2 is a generalization of a result obtained by Cheng [3] for harmonic functions of order 2.

4. Before proceeding with the proof of Theorem 1, it will be necessary to establish certain lemmas. In particular the connection between the generalized $r$ th Laplacian and the ordinary $r$ th iterated Laplacian will be established.

Lemma 1. Let $F(x, y)$ be in class $C^{(2 r)}$ on a domain $G, r \geqq 1$. If $\bar{D}\left(x_{0}, y_{0} ; t\right) \subset G$, then

$$
\begin{aligned}
A\left(F ; x_{0}, y_{0} ; t\right)= & \sum_{i=0}^{r-1} \Delta^{i} F\left(x_{0}, y_{0}\right) t^{2 i} /(i+1)\left[2^{i} i !\right]^{2} \\
& +\Delta^{r} F\left(x^{\prime}, y^{\prime}\right) t^{2 r} /(r+1)\left[2^{r} r !\right]^{2}
\end{aligned}
$$

where $\left(x^{\prime}, y^{\prime}\right)$ is in $\bar{D}\left(x_{0}, y_{0} ; t\right)$.

For by $\left[5\right.$, p. 261 , formulas $(38)$ and $\left.\left(38^{\prime}\right)\right]$,

$$
\begin{aligned}
L\left(F ; x_{0}, y_{0} ; t\right)= & \sum_{i=0}^{r-1} \Delta^{i} F\left(x_{0}, y_{0}\right) t^{2 i} /\left[2^{i} i !\right]^{2} \\
& +\iint_{D\left(x_{0}, y_{0} ; t\right)} v_{r-1}(x, y) \Delta^{r} F(x, y) d x d y
\end{aligned}
$$

where $v_{r-1}(x, y) \geqq 0$ in $D\left(x_{0}, y_{0} ; t\right)$ and is given by the recursion formulas

$$
\begin{aligned}
v_{r-1}(x, y) & =V_{r-1}(\tau) \quad \text { where } \tau=\left[\left(x-x_{0}\right)^{2}+\left(y-y_{0}\right)^{2}\right]^{1 / 2}, \\
V_{j+1}(\tau) & =\int_{\tau}^{t} \rho V_{j}(\rho) \log (\rho / \tau) d \rho, \\
V_{0}(\tau) & =(2 \pi)^{-1} \log (t / \tau) .
\end{aligned}
$$


If the function $F(x, y)$ in (4) is chosen to be $\left[\left(x-x_{0}\right)^{2}+\left(y-y_{0}\right)^{2}\right]^{r}$, then $\Delta^{i} F\left(x_{0}, y_{0}\right)=0(i=0,1,2, \cdots, r-1), \Delta^{r} F(x, y) \equiv\left[2^{r} r !\right]^{2}$, and $L\left(F ; x_{0}, y_{0} ; t\right)=t^{2 r}$, so that we obtain the identity

$$
\iint_{D\left(x_{0}, y_{0} ; t\right)} v_{r-1}(x, y) d x d y=t^{2 r} /\left[2^{r} r !\right]^{2} .
$$

Now, since $\Delta^{r} F(x, y)$ is continuous for any $F(x, y)$ satisfying the conditions of the lemma, the integral on the right side of (4) may be replaced by $\Delta^{r} F\left(x_{t}^{\prime \prime}, y_{t}^{\prime \prime}\right) t^{2 r} /\left[2^{r} r !\right]^{2}$, according to the mean-value theorem, where $\left(x_{t}^{\prime \prime}, y_{t}^{\prime \prime}\right)$ is a point of $\bar{D}\left(x_{0}, y_{0} ; t\right)$. Since

$$
A\left(F ; x_{0}, y_{0} ; t\right)=\frac{2}{t^{2}} \int_{0}^{t} \rho L\left(F ; x_{0}, y_{0} ; \rho\right) d \rho,
$$

we may multiply (4) by $t$ and integrate term-by-term to obtain

$$
A\left(F ; x_{0}, y_{0} ; t\right)=\sum_{i=0}^{r-1} \frac{\Delta^{i} F\left(x_{0}, y_{0}\right) t^{2 i}}{(i+1)\left[2^{i} i !\right]^{2}}+\frac{2}{t^{2}} \int_{0}^{t} \Delta^{r} F\left(x_{\rho}^{\prime \prime}, y_{\rho}^{\prime \prime}\right) \frac{\rho^{2 r+1}}{\left[2^{r} r !\right]^{2}} d \rho .
$$

By the mean-value theorem, the last integral may be replaced by

$$
\Delta^{r} F\left(x^{\prime}, y^{\prime}\right) t^{2 r} /(r+1)\left[2^{r} r !\right]^{2},
$$

where $\left(x^{\prime}, y^{\prime}\right)$ is some point in $\bar{D}\left(x_{0}, y_{0} ; t\right)$, so that the lemma is established.

Lemma 2. Let $F(x, y)$ be in class $C^{(2 r)}$ on a domain $G, r \geqq 0$. Then $\Delta_{2}^{\prime} F(x, y)=\Delta^{i} F(x, y)$ for $i=0, \cdots, r$.

For $r \geqq 1$, the proof of this lemma follows immediately from Lemma 1 and the continuity of $\Delta^{r} F(x, y)$. For $r=0$, the proof follows from the continuity of $F(x, y)$. It is also clear that in the conclusion of the lemma $\Delta_{2}^{t} F(x, y)$ can be replaced by $\Delta_{1}^{i} F(x, y)$.

Lemma 3. Let $F(x, y)$ be in class $C^{(2 r)}$ on a domain $G, r \geqq 0$. If $\Delta^{r} F(x, y)$ takes a maximum at $\left(x_{0}, y_{0}\right)$ in $G$ and if $\Delta_{2}^{r+1} F\left(x_{0}, y_{0}\right)$ exists, then $\Delta_{2}^{r+1} F\left(x_{0}, y_{0}\right) \leqq 0$.

For suppose the contrary were true, and let us suppose $r \geqq 1$. Then

$$
\begin{aligned}
A\left(F ; x_{0}, y_{0} ; t\right)= & \sum_{i=0}^{r} \Delta^{i} F\left(x_{0}, y_{0}\right) t^{2 i} /(i+1)\left[2^{i} i !\right]^{2} \\
& +\epsilon t^{2 r+2} /(r+2)\left[2^{r+1}(r+1) !\right]^{2}+o\left(t^{2 r+2}\right)
\end{aligned}
$$

where $\epsilon>0$ and $t$ is sufficiently small. By Lemma 1 , however, 


$$
\begin{aligned}
A\left(F ; x_{0}, y_{0} ; t\right)= & \sum_{i=0}^{r-1} \Delta^{i} F\left(x_{0}, y_{0}\right) t^{2 i} /(i+1)\left[2^{i} i !\right]^{2} \\
& +t^{2 r} \Delta^{r} F\left(x_{t}^{\prime}, y_{t}^{\prime}\right) /(r+1)\left[2^{r} r !\right]^{2}
\end{aligned}
$$

where $\left(x_{t}^{\prime}, y_{t}^{\prime}\right)$ is in $\bar{D}\left(x_{0}, y_{0} ; t\right)$.

From (5) and (6), for $t$ sufficiently small, we obtain that

$$
\Delta^{r} F\left(x_{t}^{\prime}, y_{t}^{\prime}\right)-\Delta^{r} F\left(x_{0}, y_{0}\right)=\epsilon t^{2} / 2^{2}(r+1)(r+2)+o\left(t^{2}\right),
$$

which contradicts the fact that $\Delta^{r} F(x, y)$ attains a maximum at $\left(x_{0}, y_{0}\right)$.

A similar argument prevails in case $r=0$.

5. To prove Theorem 1 , we observe that, by [1], $G-E$ is a domain. Let $\bar{D}\left(x_{0}, y_{0} ; t\right)$ be contained in $G-E$, and let $H(x, y)$ $\geqq \Delta^{r-1} F(x, y)$ on $C\left(x_{0}, y_{0} ; t\right)$, harmonic in $D\left(x_{0}, y_{0} ; t\right)$, and continuous in $\bar{D}\left(x_{0}, y_{0} ; t\right)$. Form $\Delta^{-(r-1)} H(x, y)$ and

$$
\Delta^{-(r-1)}\left[-\frac{1}{2 \pi} \iint_{D\left(x_{0}, y_{0} ; t\right)} \epsilon g(x, y ; p, q) d p d q\right]
$$

with respect to $D\left(x_{0}, y_{0} ; t\right)$, where $g(x, y ; p, q)$ is the Green's function for the disc and $\epsilon>0$. Then clearly both of the $(r-1)$ anti-Laplacians are in $C^{(\infty)}$ on the interior of the disc, and consequently, by Lemma 2 and the hypotheses of the theorem,

$$
\Delta_{2}^{r}\left\{F(x, y)-\Delta^{-(r-1)} H(x, y)\right.
$$

$$
\left.\begin{array}{l}
+\Delta^{-(r-1)} \\
>0
\end{array}\left[-\frac{1}{2 \pi} \iint_{D\left(x_{0}, y_{0} ; t\right)} \epsilon g(x, y ; p, q) d p d q\right]\right\}
$$
in the interior of $D\left(x_{0}, y_{0} ; t\right)$.

Now $\Delta^{r-1} F(x, y)-H(x, y)-(1 / 2 \pi) \iint_{D\left(x_{0}, y_{0} ; t\right)} \epsilon g(x, y ; p, q) d p d q$ is continuous in $\bar{D}\left(x_{0}, y_{0} ; t\right)$ and nonpositive on $C\left(x_{0}, y_{0} ; t\right)$. Since, by (7) and Lemma 3, this function takes its maximum on the boundary of the disc and since furthermore $\epsilon$ is arbitrary, we obtain that $\Delta^{r-1} F(x, y) \leqq H(x, y)$ throughout $\bar{D}\left(x_{0}, y_{0} ; t\right)$.

In particular, choosing $H(x, y)$ as the Dirichlet solution for $\Delta^{r-1} F(x, y)$ on $C\left(x_{0}, y_{0} ; t\right)$, we have that

$$
\begin{aligned}
\Delta^{r-1} F\left(x_{0}, y_{0}\right) & \leqq H\left(x_{0}, y_{0}\right)=L\left(H ; x_{0}, y_{0} ; t\right) \\
& =L\left(\Delta^{r-1} F(x, y) ; x_{0}, y_{0} ; t\right)
\end{aligned}
$$

from which we conclude by $[8$, p. 7$]$ that $\Delta^{r-1} F(x, y)$ is subharmonic 
in $G-E$. From the continuity of $\Delta^{r-1} F(x, y)$ in $G$ and $[2$, p. 31], the proof of the theorem then follows.

6. Corollary 1 follows immediately from Theorem 1 on recognition of the fact that $\Delta^{-1} f(x, y)$ is in $C^{(2)}$ on $R$ (see [6, p. 289]).

All but the last statement of Corollary 2 follows directly from Theorem 1, after restating the theorem for superharmonic functions, applying it then to show that $\Delta^{r-1} F(x, y)$ is harmonic in the domain $G-G E_{1}$, and then applying $[7$, p. 335 , Theorem VI] to obtain the fact that $\Delta^{r-1} F(x, y)$ is harmonic in $G$.

To prove the last statement of Corollary 2 , choose $G=D(0,0 ; 1)$ and

$$
F(x, y)= \begin{cases}x^{2(r-1)} & \text { for } x \geqq 0, \\ -x^{2(r-1)} & \text { for } x<0 .\end{cases}
$$

Then clearly $F(x, y)$ is of class $C^{2(r-1)-1}$ on $G$ and $\Delta_{2}^{r} F(x, y)=0$ for $(x, y)$ in $G$. But $\Delta^{r-1} F(x, y)$, having irremovable discontinuities in $G$, is clearly not harmonic there.

7. In conjunction with [9, Theorem 2], the results of this paper concerning subharmonic functions of order $r$ can be applied to the theory of double trigonometric series. In particular we can prove the following theorem:

THEOREM 2. Given the double trigonometric series $\sum a_{m n} e^{i(m x+n y)}$ where $a_{m n}$ are arbitrary complex numbers which are $O\left[\left(m^{2}+n^{2}\right)^{-\epsilon}\right]$, $\epsilon>0$, and are such that $a_{m n}=\bar{a}_{-m-n}$. Let $E$ be a closed set of capacity zero contained in the interior of the fundamental square $\Omega=\{(x, y) ; 0 \leqq x$ $\leqq 2 \pi, 0 \leqq y \leqq 2 \pi\}$. Suppose that the series is circularly summable $(C, 2 \alpha)$ to $L(x, y)$ in $\Omega-E$ where $2 \alpha$ is a non-negative integer and $L(x, y)$ is a finite-valued non-negative function. Let $r$ be an integer $\geqq \alpha+1$. Then the function

$$
F(x, y)=\frac{a_{00}(x+y)^{2 r}}{2^{r}[(2 r) !]}+\sum_{1 \leqq m^{2}+n^{2}}(-1)^{r} \frac{a_{m n}}{\left(m^{2}+n^{2}\right)^{r}} e^{i(m x+n y)}
$$

is subharmonic of order $r$ in the plane.

We say that the above double trigonometric series is circularly convergent to $L(x, y)$ if the circular partial sums

$$
S_{R}(x, y)=\sum_{m^{2}+n^{2} \leqq R^{2}} a_{m n} e^{i(m x+n y)}
$$

converge to $L(x, y)$. If 


$$
\sigma_{R}^{(\eta)}=\frac{2 \eta}{R^{2 \eta}} \int_{0}^{R} S_{u}(x, y)\left(R^{2}-u^{2}\right)^{\eta-1} u d u \rightarrow L(x, y) \quad \text { as } R \rightarrow \infty,
$$

where $\eta>0$, the series is said to be circularly summable $(C, \eta)$ to $L(x, y)$.

To prove the theorem, we first notice that $F(x, y)$ is in $C^{2(r-1)}$ on the plane. Second, by [9, Theorem 2] under the assumptions of the theorem, if the series is $(C, 2 \alpha)$ summable to $L\left(x_{0}, y_{0}\right)$ at the point $\left(x_{0}, y_{0}\right)$, then $\Delta_{2}^{r} F\left(x_{0}, y_{0}\right)=L\left(x_{0}, y_{0}\right) \geqq 0$. Now choose any point $\left(x_{1}, y_{1}\right)$ in the plane. Then under the assumptions of the theorem there exist a domain $G_{1}$ containing $\left(x_{1}, y_{1}\right)$ and a closed bounded set $E_{1}$ of capacity zero contained in $G_{1}$ such that $\Delta_{2}^{r} F(x, y) \geqq 0$ in $G_{1}-E_{1}$. By Theorem 1 of this paper, $F(x, y)$ is subharmonic of order $r$ in $G_{1}$. Since $\left(x_{1}, y_{1}\right)$ was an arbitrary point in the plane, the proof of the theorem is complete.

8. We close with the following application of Corollary 2 to the uniqueness theory of double trigonometric series:

Theorem 3. Let $\sum a_{m n} e^{i(m x+n y)}$ be a double trigonometric series where the $a_{m n}$ are arbitrary complex numbers which are $O\left[\left(m^{2}+n^{2}\right)^{-\epsilon}\right], \epsilon>0$. Let $E$ be a closed set of capacity zero contained in the fundamental square $\Omega$. Suppose the series is circularly summable $(C, \eta)$ to zero in $\Omega-E$, $\eta \geqq 0$. Then the series vanishes identically.

This theorem is seen to be a type of generalization of $[4$, Theorem 1] and [10, Theorem 1].

To prove the theorem, choose an integer $2 \alpha \geqq \eta$ and an integer $r \geqq \alpha+1$. Then the series is circularly summable $(C, 2 \alpha)$ to zero in $\Omega-E$. Now let $\left(x_{0}, y_{0}\right)$ be any point in the plane. Then clearly for the disc $D\left(x_{0}, y_{0} ; t\right)$, there exists a closed bounded set $E_{1}$ of capacity zero such that the series is summable $(C, 2 \alpha)$ to zero in $D\left(x_{0}, y_{0} ; t\right)$ $-D\left(x_{0}, y_{0} ; t\right) E_{1}$, and consequently by $[9$, Theorem 2$]$,

$$
\Delta_{2}^{r} F(x, y)=0
$$

in this same domain, where $F(x, y)$ has the form (8). Since $F(x, y)$ is in $C^{2(r-1)}$, we conclude by Corollary 2 of this paper that $F(x, y)$ is harmonic of order $r$ in $D\left(x_{0}, y_{0} ; t\right)$ and consequently in the whole plane. Therefore,

$$
f(x, y)=\frac{a_{00}(x+y)^{2}}{4}+\sum_{1 \leqq m^{2}+n^{2}}(-1) \frac{a_{m n}}{\left(m^{2}+n^{2}\right)} e^{i(m x+n y)}
$$

is harmonic in the whole plane. A computation then shows that 


$$
\begin{aligned}
f(x, y)= & A(f ; x, y ; t)=\frac{a_{00}}{4}\left[(x+y)^{2}+t^{2} / 2\right] \\
& -2 \sum_{1 \leqq m^{2}+n^{2}} a_{m n} e^{i(m x+n y)} \frac{J_{1}\left\lfloor\left(m^{2}+n^{2}\right)^{1 / 2} t\right]}{\left(m^{2}+n^{2}\right)^{3 / 2} t} .
\end{aligned}
$$

Fixing $(x, y)$, letting $t$ tend to infinity, and observing then that the series on the right side of (9) tends to zero, we are able to conclude first that $a_{00}=0$, then that $f(x, y)=0$, and then, consequently, that $a_{m n}=0$ for all $(m, n)$.

\section{REFERENCES}

1. M. Brelot, Sur la structure des ensembles de capacité nulle, C. R. Acad. Sci. Paris vol. 192 (1931) pp. 206-208.

2. - Etude des fonctions sousharmoniques au voisinage d'un point, Paris, 1934.

3. M. T. Cheng, On a theorem of Nicolèsco and generalized Laplace operators, Proc. Amer. Math. Soc. vol. 2 (1951) pp. 77-85.

4. - Uniqueness of multiple trigonometric series, Ann. of Math. vol. 52 (1950) pp. $403-416$.

5. R. Courant and D. Hilbert, Methoden der mathematischen Physik, vol. 2, Berlin, 1937.

6. N. M. Gunther, La theorie du potentiel et ses applications aux problèmes fondamentaux de la physique mathêmatique, Paris, 1934.

7. O. D. Kellogg, Foundation of potential theory, Berlin, 1929.

8. T. Rad6, Subharmonic functions, Ergebnisse der Mathematik, vol. 5, no. 1, Berlin, 1937.

9. V. L. Shapiro, Generalized Laplacians of the second kind and double trigonometric series, Duke Math. J. vol. 21 (1954) pp. 173-178.

10. - An extension of results in the uniqueness theory of double trigonometric series, Duke Math. J. vol. 20 (1953) pp. 359-365.

\section{RUTGERS UNIVERSITY AND}

The Institute For Advanced Study 